\title{
Gabor wavelet analysis and the fractional Hilbert transform
}

\author{
Kunal Narayan Chaudhury and Michael Unser \\ Biomedical Imaging Group, \\ Ecole Polytechnique Fédérale de Lausanne (EPFL), Switzerland
}

\begin{abstract}
We propose an amplitude-phase representation of the dual-tree complex wavelet transform (DT-CWT) which provides an intuitive interpretation of the associated complex wavelet coefficients. The representation, in particular, is based on the shifting action of the group of fractional Hilbert transforms (fHT) which allow us to extend the notion of arbitrary phase-shifts beyond pure sinusoids. We explicitly characterize this shifting action for a particular family of Gabor-like wavelets which, in effect, links the corresponding dual-tree transform with the framework of windowed-Fourier analysis.

We then extend these ideas to the bivariate DT-CWT based on certain directional extensions of the fHT. In particular, we derive a signal representation involving the superposition of direction-selective wavelets affected with appropriate phase-shifts.
\end{abstract}

Keywords: fractional Hilbert transform, dual-tree complex wavelet transform, amplitude-phase factors, Gabor wavelets analysis.

\section{INTRODUCTION}

\subsection{The dual-tree transform}

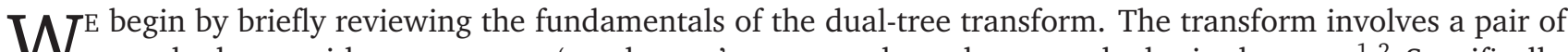
wavelet bases with a one-to-one 'quadrature' correspondence between the basis elements. ${ }^{1,2}$ Specifically, one considers a primary wavelets basis $\left\{\psi_{i, k}\right\}_{(i, k) \in \mathbf{Z}^{2}}$ of $\mathrm{L}^{2}(\mathbf{R})$ generated through the dilation-translations of a single prototype $\psi(x)$; that is, $\psi_{i, k}(x)=\Xi_{i, k} \psi(x)$ where $\Xi_{i, k} f(x)=2^{i / 2} f\left(2^{i} x-k\right)$ denotes the (normalized) dilation-translation operator corresponding to integers $i$ and $k$. The highlight of the transform is then the construction of a secondary wavelet basis $\left\{\psi_{i, k}^{\prime}\right\}_{(i, k) \in \mathbf{Z}^{2}}$ having the correspondence $\psi_{i, k}^{\prime}(x)=\mathscr{H} \psi_{i, k}(x)$, where $\mathscr{H}$ denotes the Hilbert transform (HT) operator:

$$
\mathscr{H} f(x) \stackrel{\mathscr{F}}{\longleftrightarrow}-j \operatorname{sign}(\omega) \hat{f}(\omega) .
$$

The HT acts as a quadrature transform that takes $\cos \left(\omega_{0} x\right)$ into $\sin \left(\omega_{0} x\right)$, and as an orthogonal transform on $\mathrm{L}^{2}(\mathbf{R})$ in the sense that $\langle f, \mathscr{H} f\rangle=0$ for all $f(x)$ in this space. Though this is not at all obvious a priori, it turns out (as suggested by the notation) that the secondary wavelet basis can also be realized through the dilationstransations of the HT counterpart $\psi^{\prime}(x)=\mathscr{H} \psi(x)$. This, in fact, is possible thanks to certain fundamental invariances enjoyed by the HT operator. In particular, following definition (1), one can readily verify that the HT commutes with translations and dilations; in particular,

$$
\mathscr{H} \Xi_{i, k}=\Xi_{i, k} \mathscr{H}
$$

and that it is unitary:

$$
\|\mathscr{H} f\|_{\mathrm{L}^{2}}=\|f\|_{\mathrm{L}^{2}} \quad\left(f \in \mathrm{L}^{2}(\mathbf{R})\right) .
$$

It is then easily deduced that the functions $\psi_{i, k}^{\prime}(x)=\Xi_{i, k} \psi^{\prime}(x)$ indeed constitute a wavelet basis of $\mathrm{L}^{2}(\mathbf{R})$, and that the correspondence $\psi_{i, k}^{\prime}(x)=\mathscr{H} \psi_{i, k}(x)$ holds for every integer $i$ and $k .^{3}$

Correspondence: \{kunal.chaudhury, michael.unser\}@epfl.ch. This work was supported by the Swiss National Science Foundation under grant 200020-109415.

Wavelets XIII, edited by Vivek K. Goyal, Manos Papadakis, Dimitri Van De Ville, Proc. of SPIE Vol. 7446, 74460T · @ 2009 SPIE · CCC code: 0277-786X/09/\$18 · doi: 10.1117/12.824863 
The application of the transform involves the simultaneous analysis of a signal $f(x)$ in $\mathrm{L}^{2}(\mathbf{R})$ in terms of the quadrature wavelet bases $\left\{\psi_{i, k}\right\}$ and $\left\{\psi_{i, k}^{\prime}\right\}$. In particular, one considers the wavelet expansions

$$
f(x)=\left\{\begin{array}{l}
\sum_{(i, k) \in \mathbf{Z}^{2}} a_{i}[k] \psi_{i, k}(x), \\
\sum_{(i, k) \in \mathbf{Z}^{2}} b_{i}[k] \psi_{i, k}^{\prime}(x),
\end{array}\right.
$$

where the expansion coefficients in (4) are specified by the dual wavelet bases $\left\{\tilde{\psi}_{i, k}\right\}$ and $\left\{\tilde{\psi}_{i, k}^{\prime}\right\}$ through the projections

$$
a_{i}[k]=\left\langle f, \tilde{\psi}_{i, k}\right\rangle, \quad \text { and } \quad b_{i}[k]=\left\langle f, \tilde{\psi}_{i, k}^{\prime}\right\rangle .
$$

In effect, this allows one to identify the complex wavelet coefficients $c_{i}[k]=\left(a_{i}[k]+j b_{i}[k]\right) / 2$, and the associated amplitude-phase factors $\left|c_{i}[k]\right|$ and $\arg \left(c_{i}[k]\right.$ ) (the use of the factor $1 / 2$ will be justified shortly). As a consequence of (2) and (3), the dual wavelet bases can also be generated through the dilations-translations of two dual wavelets, $\tilde{\psi}(x)$ and $\tilde{\psi}^{\prime}(x)$, that form a HT pair as well.

\subsection{Multiresolution Gabor-like transforms}

A framework for constructing HT-pairs of wavelets (within Mallat's multiresolution framework) was recently proposed based on a spectral factorization result for scaling functions. ${ }^{3}$ In particular, it was shown that a multiresolution form of Gabor-like analysis could be achieved within the framework of the dual-tree transform. This was founded on the two vital observations. The first one was that the extended $(\alpha, \tau)$ family of B-spline wavelets $\psi(x ; \alpha)$ (indexed by the approximation order $\alpha+1$ ) is closed with respect to the action of the HT operator (the corresponding discrete wavelet transform has an efficient FFT-based implementation). Secondly, it was shown that the complex spline wavelet $\Psi(x)=\psi(x ; \alpha)+j \mathscr{H} \psi(x ; \alpha)$ asymptotically converges to a Gabor function:

$$
\Psi(x ; \alpha) \sim \varphi(x) \exp \left(j \omega_{0} x+\xi_{0}\right) \quad(\alpha \rightarrow+\infty),
$$

where $\varphi(x)$ is a Gaussian window, and $\omega_{0}, \xi_{0}$ are appropriate modulation parameters. As a consequence, a Gabor-like transform, involving the computation of the sequence of projections

$$
f(x) \mapsto \frac{1}{2}\left\langle f(x), \Xi_{i, k} \Psi(x ; \alpha)\right\rangle \quad(i, k \in \mathbf{Z})
$$

with the dilates-translates of the Gabor-like wavelet $\Psi(x ; \alpha)$, could be realized using the dual-tree transform corresponding to the spline wavelets $\psi(x ; \alpha)$ and $\mathscr{H} \psi(x ; \alpha)$ (sufficiently large $\alpha$ ). These ideas were also extended for the realization of a direction-selective Gabor-like transform.

\subsection{Present Contribution}

In this paper, we provide a characterization of the dual-tree transform, and the Gabor-like transforms in particular, from the perspective of multiresolution windowed-Fourier analysis. In particular, we link the multiresolution wavelet-framework of the former with the intuitive amplitude-phase representation associated with the latter. Complex wavelets, derived from the combination of non-redundant wavelet bases, provide an attractive means of encoding the relative signal "displacements" using the phase relation between the components. The DT-CWT is a particular instance where the components are related through the HT.

In $\S 2$, we derive a representation of the dual-tree transform using the group of fractional Hilbert transform (fHT) operators:

$$
\mathcal{H}_{\tau}=\cos (\pi \tau) \mathscr{I}-\sin (\pi \tau) \mathscr{H} \quad(\tau \in \mathbf{R})
$$

( $\mathscr{I}$ is the identity operator). In particular, we are able to interpret the phase factors associated with the dualtree transform in terms of the action of this group. However, it is the fundamental invariances (2) and (3) inherited by this extended family of operators that play a decisive role in establishing the windowed-Fourier-like representation (cf. (11)).

The proposed windowed Fourier-like representation admits a straightforward extension to the bivariate setting by introducing an appropriate multi-dimensional extensions of the HT. In particular, we arrive at a representation (cf. (15)) involving the superposition of the direction-selective synthesis wavelets affected with appropriate phase-shifts. This provides an explicit understanding of the phase-shift action of the fdHT operators for a the particular family of 2D Gabor-like wavelets derived through the tensor products of 1D Gabor-like wavelets. 


\section{DUAL-TREE GABOR WAVELET ANALYSIS}

\subsection{Signal representation: interpretation of the amplitude-phase factors}

Our objective is to derive a representation of $f(x)$ in terms of the amplitude-phase factors $c_{i}[k]=\left|c_{i}[k]\right| \mathrm{e}^{j \phi_{i}[k]}$. Clearly, the transformation

$$
f(x) \mapsto\left\{c_{i}[k]\right\}_{(i, k) \in \mathbf{Z}^{2}}
$$

constitutes a overcomplete representation of $f(x)$. In particular, given the coefficients $c_{i}[k]$, there exists nonunique ways of reconstructing the input $f(x)$. We consider the simplest inversion procedure involving the inversion of both the forward transforms, as in (4), followed by the averaging of the reconstructed signals. In particular, by combining the expansions in (4) and by invoking the dilation-translation invariance of the fHTs, we arrive at the following representation:

$$
\begin{aligned}
f(x) & =\frac{1}{2} \sum_{(i, k) \in \mathbf{Z}^{2}}\left(a_{i}[k] \psi_{i, k}(x)+b_{i}[k] \psi_{i, k}^{\prime}(x)\right) \\
& =\sum_{(i, k) \in \mathbf{Z}^{2}}\left|c_{i}[k]\right| \mathcal{H}_{\phi_{i}[k] / \pi}\left\{\psi_{i, k}(x)\right\} \\
& =\sum_{(i, k) \in \mathbf{Z}^{2}}\left|c_{i}[k]\right| \Xi_{i, k}\left\{\psi\left(x ; \tau_{i}[k]\right)\right\} .
\end{aligned}
$$

Here the synthesis wavelet $\psi\left(x ; \tau_{i}[k]\right)$ is derived from the mother wavelet $\psi(x)$ through the action the fHT corresponding to the shift $\tau_{i}[k]=\phi_{i}[k] / \pi$. The unitary nature of the fHT ensures that these fractionally-shifted synthesis wavelets have identical norms. In particular, while the amplitude $\left|c_{i}[k]\right|$ indicates the strength of wavelet correlation, the local signal displacement gets encoded in the shift $\tau_{i}[k]$ which specifies the most "appropriate" wavelet within the family $\left\{\mathcal{H}_{\tau} \psi_{i, k}\right\}_{\tau \in \mathbf{R}}$.

\subsection{Characterization of the Gabor-like transform}

It turns out that the shifted wavelets $\psi\left(x ; \tau_{i}[k]\right)$ in (8) can be explicitly characterized when $\psi(x)$ is a (real) Gabor-wavelet,

$$
\psi(x)=\varphi(x) \cos \left(\omega_{0} x+\xi_{0}\right) .
$$

This formula describes the asymptotic form of the Gabor-like wavelet $\Psi(x ; \alpha)$ in (6). For reasons that will be evident shortly, we choose to flip the roles of the analysis and synthesis wavelets: we will analyze the signal using the dual complex wavelet $\tilde{\Psi}(x ; \alpha)=\tilde{\psi}(x ; \alpha)+j \tilde{\psi}^{\prime}(x ; \alpha)$, while the Gabor-like wavelet $\Psi(x ; \alpha)$ will be used for reconstruction.

If the window function $\varphi(x)$ is bandlimited to $[-\Omega, \Omega]$ with $\Omega<\omega_{0}$, we can make precise statements on the dual-tree representation in (8). To do so, we will need the following result:

Proposition 2.1. Let $\varphi(x)$ in (9) be bandlimited to $\left(-\omega_{0}, \omega_{0}\right)$. Then

$$
\mathcal{H}_{\tau}\left\{\varphi(x) \cos \left(\omega_{0} x\right)\right\}=\varphi(x) \cos \left(\omega_{0} x+\pi \tau\right) .
$$

That is, the fHT acts on the phase of the modulating sinusoid while preserving the Gaussian envelope. In particular, we can then rewrite (8) as

$$
f(x)=\sum_{(i, k) \in \mathbf{Z}^{2}} \overbrace{\varphi_{i, k}(x)}^{\text {fixed window }} \Xi_{i, k}\{\overbrace{\left|c_{i}[k]\right| \cos \left(\omega_{0} x+\xi_{0}+\pi \tau_{i}[k]\right)}^{\text {variable amp-phase oscillation }}\}
$$

where $\varphi_{i, k}(x)=\Xi_{i, k} \varphi(x)$ denotes the (fixed) Gaussian-like window at scale $i$ and translation $k$. This provides an explicit interpretation of the parameter $\tau_{i}[k]$ as the phase-shift applied to the modulating sinusoid of the wavelet. In effect, the oscillation is shifted to best fit the underlying signal singularities/transitions while the localization window $\varphi_{i, k}(x)$ is kept fixed. In this light, one can interpret the associated dual-tree analysis as a multiresolution 
form of the windowed-Fourier analysis, with the fundamental difference that, instead of analyzing the signal at different frequencies, it resolves the signal over different scales (or resolutions).

Figure 1 shows quadrature pairs $\left(\mathcal{H}_{\tau} \psi(x ; \alpha), \mathcal{H}_{\tau+1 / 2} \psi(x ; \alpha)\right)$ of Gabor-like spline wavelets corresponding to different $\tau$. Each of the pairs are localized within a common Gaussian-like window, and the modulating oscillations are driven to a relative quadrature through the action of the pair $\left(\mathcal{H}_{\tau}, \mathcal{H}_{\tau+1 / 2}\right)$.

\section{BIVARIATE EXTENSION}

The amplitude-phase representation derived in $\S 2$ can also be extended to the $2 \mathrm{D}$ setting where the dual-tree wavelets exhibit better directional selectivity than the conventional tensor-product (separable) wavelets. ${ }^{4}$

\subsection{Directional HT pairs of wavelets}

We briefly recall the construction framework for the bivariate DT-CWT based on the tensor-products of onedimensional analytic wavelets. ${ }^{3}$ Specifically, let $\varphi(x)$ and $\varphi^{\prime}(x)$ denote the scaling functions associated with the analytic wavelet $\psi_{a}(x)=\psi(x)+j \psi^{\prime}(x)$, where $\psi^{\prime}(x)=\mathscr{H} \psi(x)$. The 2D dual-tree construction then hinges on the identification of four separable multiresolutions of $\mathrm{L}^{2}\left(\mathbf{R}^{2}\right)$ that are naturally associated with the two scaling functions: the approximation subspaces $V(\varphi) \otimes V(\varphi), V(\varphi) \otimes V\left(\varphi^{\prime}\right), V\left(\varphi^{\prime}\right) \otimes V(\varphi)$ and $V\left(\varphi^{\prime}\right) \otimes V\left(\varphi^{\prime}\right)$, and their multiscale counterparts. The corresponding separable wavelets - the 'low-high', 'high-low' and 'high-high' wavelets - are specified by:

$$
\begin{array}{ll}
\bar{\psi}_{1}(\boldsymbol{x})=\varphi(x) \psi(y), & \bar{\psi}_{4}(\boldsymbol{x})=\varphi(x) \psi^{\prime}(y), \\
\bar{\psi}_{2}(\boldsymbol{x})=\psi(x) \varphi(y), & \bar{\psi}_{5}(\boldsymbol{x})=\psi(x) \varphi^{\prime}(y), \\
\bar{\psi}_{3}(\boldsymbol{x})=\psi(x) \psi(y), & \bar{\psi}_{6}(\boldsymbol{x})=\psi(x) \psi^{\prime}(y), \\
\bar{\psi}_{7}(\boldsymbol{x})=\varphi^{\prime}(x) \psi(y), & \bar{\psi}_{10}(\boldsymbol{x})=\varphi^{\prime}(x) \psi^{\prime}(y), \\
\bar{\psi}_{8}(\boldsymbol{x})=\psi^{\prime}(x) \varphi(y), & \bar{\psi}_{11}(\boldsymbol{x})=\psi^{\prime}(x) \varphi^{\prime}(y), \\
\bar{\psi}_{9}(\boldsymbol{x})=\psi^{\prime}(x) \psi(y), & \bar{\psi}_{12}(\boldsymbol{x})=\psi^{\prime}(x) \psi^{\prime}(y) .
\end{array}
$$

The dual wavelets $\tilde{\bar{\psi}}_{1}(\boldsymbol{x}), \ldots, \tilde{\bar{\psi}}_{12}(\boldsymbol{x})$ are similarly defined in terms of $\tilde{\psi}(x)$ and $\tilde{\psi}^{\prime}(x)$ (here $\boldsymbol{x}=(x, y)$ denotes the planar coordinates). As far as the identification of the complex wavelets is concerned, the main issue is the poor directional selectivity of the 'high-high' wavelets along the diagonal directions. This problem can, however, be mitigated by appropriately exploiting the one-sided spectrum of the analytic wavelet $\psi_{a}(x)$, and, in effect, by appropriately combining the wavelets in (12). In particular, the complex wavelets specified by

$$
\begin{aligned}
& \Psi_{1}(\boldsymbol{x})=\psi_{a}(x) \varphi(y)=\bar{\psi}_{2}(\boldsymbol{x})+j \bar{\psi}_{8}(\boldsymbol{x}), \\
& \Psi_{2}(\boldsymbol{x})=\psi_{a}(x) \varphi^{\prime}(y)=\bar{\psi}_{5}(\boldsymbol{x})+j \bar{\psi}_{11}(\boldsymbol{x}), \\
& \Psi_{3}(\boldsymbol{x})=\varphi(x) \psi_{a}(y)=\bar{\psi}_{1}(\boldsymbol{x})+j \bar{\psi}_{4}(\boldsymbol{x}), \\
& \Psi_{4}(\boldsymbol{x})=\varphi^{\prime}(x) \psi_{a}(y)=\bar{\psi}_{7}(\boldsymbol{x})+j \bar{\psi}_{10}(\boldsymbol{x}), \\
& \Psi_{5}(\boldsymbol{x})=\frac{1}{\sqrt{2}} \psi_{a}(x) \psi_{a}(y)=\left(\frac{\bar{\psi}_{3}(\boldsymbol{x})-\bar{\psi}_{12}(\boldsymbol{x})}{\sqrt{2}}\right)+j\left(\frac{\bar{\psi}_{6}(\boldsymbol{x})+\bar{\psi}_{9}(\boldsymbol{x})}{\sqrt{2}}\right), \\
& \Psi_{6}(\boldsymbol{x})=\frac{1}{\sqrt{2}} \psi_{a}^{*}(x) \psi_{a}(y)=\left(\frac{\bar{\psi}_{3}(\boldsymbol{x})+\bar{\psi}_{12}(\boldsymbol{x})}{\sqrt{2}}\right)+j\left(\frac{\bar{\psi}_{6}(\boldsymbol{x})-\bar{\psi}_{9}(\boldsymbol{x})}{\sqrt{2}}\right),
\end{aligned}
$$

exhibit the desired directional selectivity along the primal orientations $\theta_{1}=\theta_{2}=0, \theta_{3}=\theta_{4}=\pi / 2, \theta_{5}=\pi / 4$, and $\theta_{6}=3 \pi / 4$, respectively. ${ }^{3}$ The dual complex wavelets $\tilde{\Psi}_{1}(\boldsymbol{x}), \ldots, \tilde{\Psi}_{6}(\boldsymbol{x})$ are specified in an identical fashion using the dual wavelets $\tilde{\bar{\psi}}_{p}(\boldsymbol{x})$, and are oriented along the same set of directions.

Akin to the HT correspondence, the complex wavelet components are related through the directional HT $(\mathrm{dHT})$ :

$$
\mathscr{H}_{\theta} f(\boldsymbol{x}) \stackrel{\mathscr{F}}{\longleftrightarrow}-j \operatorname{sign}\left(\boldsymbol{u}_{\theta}^{T} \boldsymbol{\omega}\right) \hat{f}(\boldsymbol{\omega}) \quad(0 \leqslant \theta<\pi),
$$




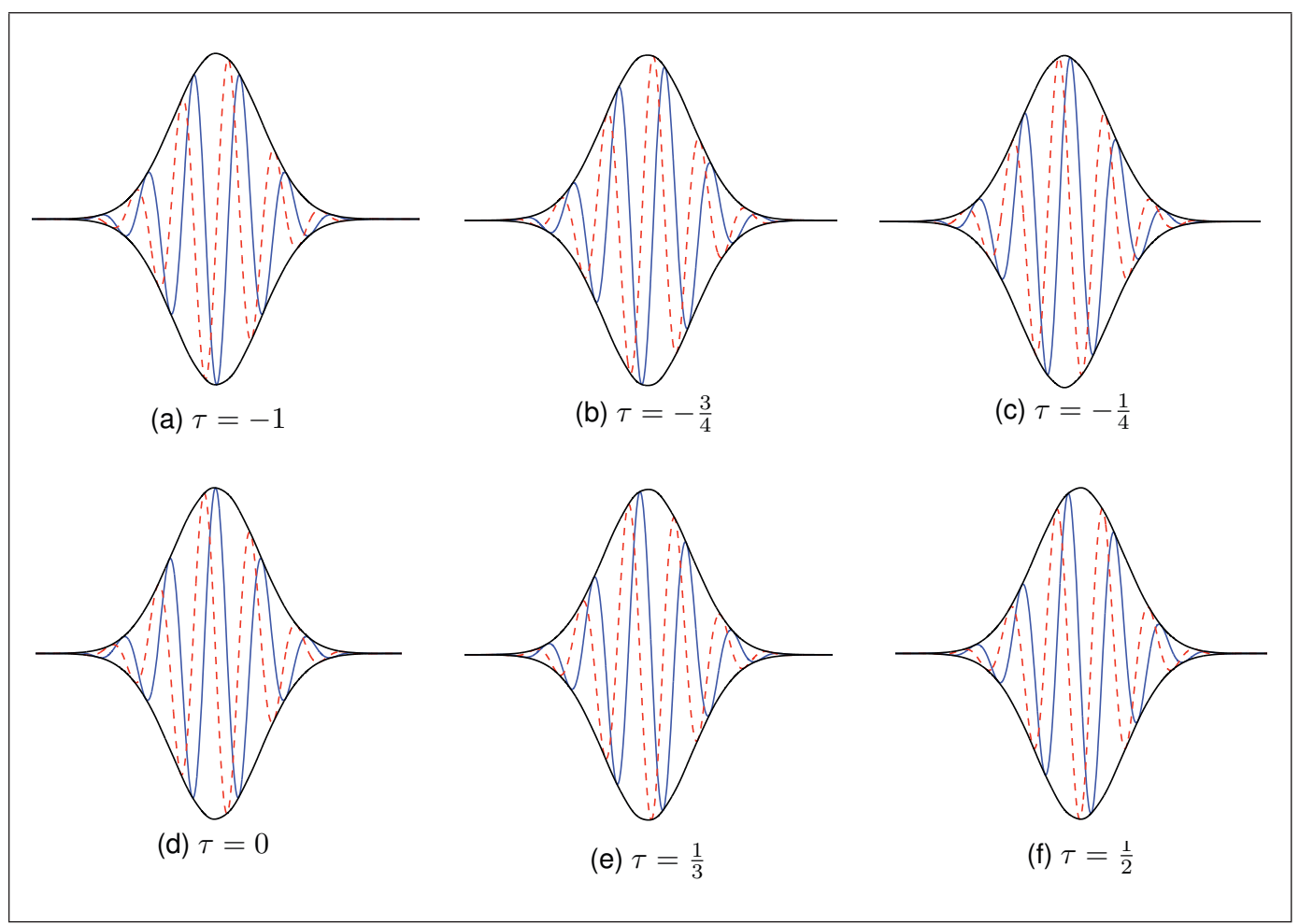

Figure 1. Quadrature pairs of Gabor-like spline wavelets obtained by the action of fHT group. Blue (solid line): $\mathcal{H}_{\tau} \psi(x ; 8)$, Red (broken line): $\mathcal{H}_{\tau+\frac{1}{2}} \psi(x ; 8)$, and Black (solid line): Common localization window given by $\left|\mathcal{H}_{\tau} \psi(x ; 8)+j \mathcal{H}_{\tau+\frac{1}{2}} \psi(x ; 8)\right|$ (http://dx.doi.org/10.1117/12.824863.1).

where $\boldsymbol{u}_{\theta}=(\cos \theta, \sin \theta)$ denotes the unit vector along the direction $\theta$. In particular, we have the correspondences

$$
\mathfrak{I m}\left(\Psi_{\ell}\right)=\mathscr{H}_{\theta_{\ell}} \mathfrak{R e}\left(\Psi_{\ell}\right) \quad(\ell=1, \ldots, 6),
$$

so that, by denoting the real component of the complex wavelet $\Psi_{\ell}(\boldsymbol{x})$ by $\psi_{\ell}(\boldsymbol{x})$, we have the convenient representation $\Psi_{\ell}(\boldsymbol{x})=\psi_{\ell}(\boldsymbol{x})+j \mathscr{H}_{\theta_{\ell}} \psi_{\ell}(\boldsymbol{x})$ that is reminiscent of the 1D analytic representation.

\subsection{Directional amplitude-phase representation}

Let us denote the dilated-translated copies of the each of the six analysis wavelets $\tilde{\Psi}_{\ell}(\boldsymbol{x})$ by $\tilde{\Psi}_{\ell, i, \boldsymbol{k}}(\boldsymbol{x})$, so that

$$
\tilde{\Psi}_{\ell, i, \boldsymbol{k}}(\boldsymbol{x})=\Xi_{i, \boldsymbol{k}} \tilde{\Psi}_{\ell}(\boldsymbol{x}) \quad\left(i \in \mathbf{Z}, \boldsymbol{k} \in \mathbf{Z}^{2}\right),
$$

where $\Xi_{i, \boldsymbol{k}}$ is specified by $\Xi_{i, \boldsymbol{k}} f(\boldsymbol{x})=2^{i} f\left(2^{i} \boldsymbol{x}-\boldsymbol{k}\right)$. The corresponding dual-tree transform involves the analysis of a finite-energy signal $f(\boldsymbol{x})$ in terms of the sequence of projections

$$
c_{i}^{\ell}[\boldsymbol{k}]=\frac{1}{4}\left\langle f, \tilde{\Psi}_{\ell, i, \boldsymbol{k}}\right\rangle .
$$

The representation of $f(\boldsymbol{x})$ in terms of the analysis coefficients $c_{i}^{\ell}[\boldsymbol{k}]$ is based on the following fractional extension of the directional HT operator:

$$
\mathcal{H}_{\theta, \tau}=\cos (\pi \tau) \mathscr{I}-\sin (\pi \tau) \mathscr{H}_{\theta} \quad(\tau \in \mathbf{R})
$$

These operators allow us to capture the notion of direction-selective phase-shifts. The key properties of the fHT, which played a decisive role in establishing the representation for the 1D counterpart, carry over directly to the 
fractional directional HT (fdHT) operators: they are invariant to translations and dilations and are unitary. In particular, based on the above properties, we derive the representation

$$
f(\boldsymbol{x})=\sum_{(\ell, i, \boldsymbol{k})}\left|c_{i}^{\ell}[\boldsymbol{k}]\right| \Xi_{i, \boldsymbol{k}}\left\{\psi_{\ell}\left(\boldsymbol{x} ; \tau_{i}^{\ell}[\boldsymbol{k}]\right)\right\}
$$

involving the superposition of direction-selective synthesis wavelets affected with appropriate phase-shifts. ${ }^{5}$ The wavelets $\psi_{\ell}\left(\boldsymbol{x} ; \tau_{i}^{\ell}[\boldsymbol{k}]\right)$ are derived from the reference wavelet $\psi_{\ell}(\boldsymbol{x})$ through the action of fdHT, corresponding to the direction $\theta_{\ell}$ and shift $\tau_{i}^{\ell}[\boldsymbol{k}]=\arg \left(c_{i}^{\ell}[\boldsymbol{k}]\right) / \pi$ As in the 1D setting, further insight into the above representation is obtained by considering wavelets resembling windowed plane waves.

\subsection{Directional Gabor-like analysis}

Akin to the 1D setting, further insight into the above representation is obtained by considering wavelets resembling windowed plane waves. A distinctive feature of the dHT, that comes as a direct consequence of (14), is its phase-shift action in relation to plane-waves: it transforms the directional $\operatorname{cosine} \cos \left(\boldsymbol{u}_{\theta}^{T} \boldsymbol{x}\right)$ into the directional $\operatorname{sine} \sin \left(\boldsymbol{u}_{\theta}^{T} \boldsymbol{x}\right)$. Moreover, what turns out to be even more crucial in the current context, is that the above action is preserved for windowed plane waves of the form

$$
\varphi(\boldsymbol{x}) \cos \left(\Omega \boldsymbol{u}_{\theta}^{T} \boldsymbol{x}\right) .
$$

In particular, as a straightforward directional extension of (10), we have the following generalization for the fractional extensions:

PROPOSITION 3.1. Suppose that $\varphi(\boldsymbol{x})$ in (16) is bandlimited to the disk $\{\boldsymbol{\omega}:\|\boldsymbol{\omega}\|<\Omega\}$. Then we have that

$$
\mathcal{H}_{\theta, \tau}\left\{\varphi(\boldsymbol{x}) \cos \left(\Omega \boldsymbol{u}_{\theta}^{T} \boldsymbol{x}\right)\right\}=\varphi(\boldsymbol{x}) \sin \left(\Omega \boldsymbol{u}_{\theta}^{T} \boldsymbol{x}+\pi \tau\right) .
$$

Thus, the fdHT acts only on the phase of the oscillation while the window remains fixed. In particular, if the dual-tree wavelets are of the form $\psi_{\ell}(\boldsymbol{x})=\varphi_{\ell}(\boldsymbol{x}) \cos \left(\Omega_{\ell} \boldsymbol{u}_{\theta_{\ell}}^{T} \boldsymbol{x}\right)$, we can then rewrite (15) as

$$
f(\boldsymbol{x})=\sum_{(\ell, i, \boldsymbol{k})} \overbrace{\varphi_{\ell, i, \boldsymbol{k}}(\boldsymbol{x})}^{\text {fixed window }} \Xi_{i, \boldsymbol{k}}\{\overbrace{\left|c_{i}^{\ell}[\boldsymbol{k}]\right| \cos \left(\Omega_{\ell} \boldsymbol{u}_{\theta_{\ell}}^{T} \boldsymbol{x}+\pi \tau_{i}^{\ell}[\boldsymbol{k}]\right)}^{\text {variable amp-phase directional wave }}\},
$$

where $\varphi_{\ell, i, \boldsymbol{k}}(\boldsymbol{x})$ represent the dilated-translated copies of $\varphi_{\ell}(\boldsymbol{x})$. The above representation explicitly highlights the role of $\tau_{i}^{\ell}[\boldsymbol{k}]$ as a "scale-dependent" measure of the local signal displacements along certain preferential directions. This is the scenario for the spline-based Gabor-like transforms ${ }^{3}$ where the dual-tree wavelets asymptotically converge to directional Gabor functions.

\section{CONCLUDING REMARKS}

We presented an amplitude-phase representation of the dual-tree transform in general, and a windowed-Fouierlike characterization of the Gabor-like transforms in particular. The signal representation was centered around one crucial construction, namely the HT correspondence between the wavelet bases. Indeed, the identification of the fHT-transformed wavelets in (11) followed as a direct consequence of this particular relation; the subsequent developments were then based on two crucial properties of the fHT, namely

- its intrinsic invariances with respect to translations, dilations and norm-evaluations, and

- its particular phase-shifting action on the Gabor wavelet.

These observations could be of potential interest in applications involving the dual-tree transform, particularly signal denoising, where a rigorous mathematical model linking the reconstructed signal to the processed complex wavelet coefficients is desirable. 


\section{Appendix: Proof of Proposition 2.1}

Proof. The result follows directly from definition (7) and the following action of the HT:

$$
\mathscr{H}\left\{\varphi(x) \cos \left(\omega_{0} x\right)\right\}=\varphi(x) \sin \left(\omega_{0} x\right) .
$$

Indeed, we see that

$$
\begin{aligned}
\mathcal{H}_{\tau}\left\{\varphi(x) \cos \left(\omega_{0} x\right)\right\} & =\cos (\pi \tau) \varphi(x) \cos \left(\omega_{0} x\right)-\sin (\pi \tau) \mathscr{H}\left\{\varphi(x) \cos \left(\omega_{0} x\right)\right\} \\
& =\cos (\pi \tau) \varphi(x) \cos \left(\omega_{0} x\right)-\sin (\pi \tau) \varphi(x) \sin \left(\omega_{0} x\right) \\
& =\varphi(x) \cos \left(\omega_{0} x+\pi \tau\right) .
\end{aligned}
$$

To establish (19), we note that the Fourier transform* of $\varphi(x) \cos \left(\omega_{0} x\right)$ is given by $\pi\left(\hat{\varphi}\left(\omega-\omega_{0}\right)+\hat{\varphi}\left(\omega+\omega_{0}\right)\right)$. Following definition (1), we then have that

$$
\begin{aligned}
& \mathscr{H}\left\{\varphi(x) \cos \left(\omega_{0} x\right)\right\} \stackrel{\mathscr{F}}{\longleftrightarrow}-j \operatorname{sign}(\omega) \cdot \pi\left(\hat{\varphi}\left(\omega-\omega_{0}\right)+\hat{\varphi}\left(\omega+\omega_{0}\right)\right) \\
&=-j \pi\left(\hat{\varphi}\left(\omega-\omega_{0}\right)-\hat{\varphi}\left(\omega+\omega_{0}\right)\right) \\
& \stackrel{\mathscr{F}}{\longleftrightarrow} \varphi(x) \sin \left(\omega_{0} x\right),
\end{aligned}
$$

since $-j \pi\left(\hat{\varphi}\left(\omega-\omega_{0}\right)-\hat{\varphi}\left(\omega+\omega_{0}\right)\right)$ is the Fourier transform of $\varphi(x) \sin \left(\omega_{0} x\right)$. Note that, in going from the first to the second step, we have used the crucial fact that the supports of $\varphi\left(\omega+\omega_{0}\right)$ and $\varphi\left(\omega-\omega_{0}\right)$ are entirely restricted to the half-lines $\{\omega<0\}$ and $\{\omega>0\}$, respectively.

\section{REFERENCES}

[1] Kingsbury, N. G., "Complex wavelets for shift invariant analysis and filtering of signals," Journal of Applied and Computational Harmonic Analysis 10, 234-253 (May 2001).

[2] Selesnick, I. W., "Hilbert transform pairs of wavelet bases," IEEE Signal Process. Lett. 8(6), 170-173 (2001).

[3] Chaudhury, K. N. and Unser, M., "Construction of Hilbert transform pairs of wavelet bases and Gabor-like transforms," IEEE Trans. on Signal Processing, in press.

[4] Selesnick, I. W., Baraniuk, R. G., and Kingsbury, N. C., "The dual-tree complex wavelet transform," IEEE Sig. Proc. Magazine 22, 123-151 (November 2005).

[5] Chaudhury, K. N. and Unser, M., "On the shiftability of dual-tree complex wavelet transforms," IEEE Trans. on Signal Processing, in press.

${ }^{*}$ we use $\hat{f}(\omega)=\int_{\mathbf{R}} f(x) \exp (-j \omega x) \mathrm{d} x$ as the definition of the Fourier transform of $f(x)$. 